\title{
Fast-and-fit vaccines
}

\author{
Results from the early trials of vaccine candidates against SARS-CoV-2 signal the fitness of vaccine technologies \\ soon to be tested at scale.
}

$\Lambda$ chieving herd immunity via effective vaccination is the only sure route out of the COVID-19 pandemic. For this reason, over two hundred vaccine-development programmes against severe acute respiratory syndrome coronavirus 2 (SARS-CoV-2) have been launched around the world since the virus' RNA was sequenced, which has enabled the synthesis of viral proteins and genomic sequences needed for vaccine development. Among the candidate vaccines, it is notable that several biotechnology companies (with support from global partnerships between public, private, philanthropic and civil society organizations) are betting on mRNA vaccines, which, although never tested in humans at scale, are expected to have shorter development and production times than traditional vaccines, and lead to faster scale-up to the many billions of doses that will be eventually needed. They are also more amenable to subsequent tweaking to improve vaccine effectiveness and safety.

Immunization with a messenger RNA sequence coding for viral antigens triggers immune responses to the injected RNA without the need for adjuvants to 'awaken' the immune system to the antigen, which, for nucleic-acid vaccines, is produced and presented by the cells that have taken up the injected sequence. Classical vaccination instead involves the injection of the virus itself, attenuated or inactivated, or of viral antigens as purified proteins (so-called 'subunit vaccines'). But recombinant proteins are expensive to manufacture at scale and require the addition of adjuvants. For SARS-CoV-2, whether nucleic-acid vaccines, live-attenuated vaccines, inactivated vaccines or subunit vaccines (particularly those using the spike protein of SARS-CoV-2) will prevent infections or lessen the severity of COVID-19 with the least or less severe adverse events, will only be determined in upcoming phase III trials.

Years of preclinical work with experimental models of infection have shown that mRNA vaccines can trigger the production of neutralizing antibodies and be efficacious prophylactically and therapeutically ${ }^{1}$. Two mRNA vaccine formulations against COVID-19 have now been tested in tens of volunteers: one developed by a collaboration between Pfizer and BioNTech ${ }^{2,3}$, and the other by

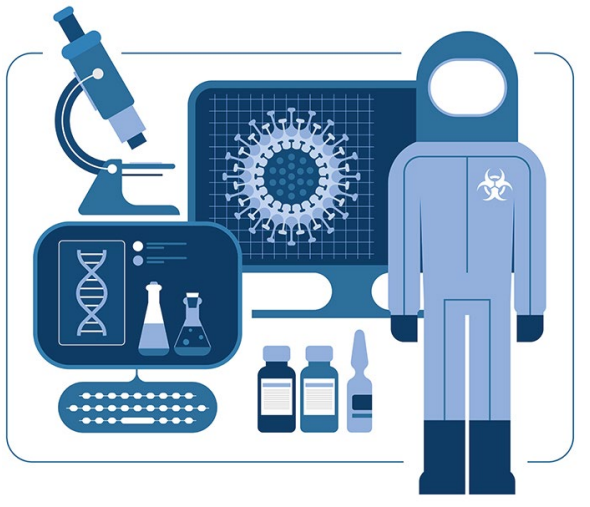

Credit: Danjazzia / Alamy Stock Vector

Moderna and the National Institute of Allergy and Infectious Diseases (NIAID) in the United States ${ }^{4}$. Both formulations use lipid nanoparticles encapsulating nucleoside-modified mRNA coding for a trimerized receptor-binding domain of the spike protein (Pfizer/BioNTech) or for its S2 subunit (Moderna/NIAID). Other differences between the two formulations lie in the particular nucleoside modifications that increase the translation of the mRNA and decrease the immune responses to it, rather than to the antigen that it encodes. The preliminary results reported so far include assessments of the elicited immune responses and of adverse events. Both vaccine candidates led to the production of spike-protein-specific $\mathrm{T}$ cells (both $\mathrm{CD} 4^{+}$ and $\mathrm{CD}^{+}$) and neutralizing antibodies, which, after a booster shot, reached levels comparable to those raised by patients recovering from COVID-19. Safety-wise, the vaccines caused transient and mild adverse effects - such as pain at the injection site and flu-like symptoms - that are common to many vaccines. Notably, the neutralizing antibodies raised by the Pfizer/BioNTech vaccine were also active in vitro against 16 mutated versions of the spike protein's receptor-binding domain and a dominant circulating variant (D614G) of the spike protein ${ }^{3}$.

Other leading vaccine candidates use replication-deficient adenoviruses in which the genetic material incorporates DNA coding for the spike protein of SARS-CoV-2. These vaccines are developed by the University of Oxford in partnership with
AstraZeneca (which uses a chimpanzee adenovirus $^{5}$ ), and by CanSino Biologics (which uses a human adenovirus ${ }^{6}$ ). Such DNA vaccines using recombinant adenoviruses have also shown encouraging safety and immune-response profiles in hundreds of volunteers. Adenoviral vectors are strongly immunogenic, which has both advantages and disadvantages: on one hand, a highly immunogenic formulation is more likely to produce a strong immune response to the viral antigen; on the other hand, adenoviruses trigger an immune response to the vector itself, which can significantly reduce the effectiveness of subsequent boosting immunizations. The effectiveness of first immunizations is also reduced in people with pre-existing immunity to the viral vector, as is the case for the CanSino formulation, whose adenovirus was used in the Ebola vaccine approved by the Chinese Food and Drug Administration in 2017. No DNA vaccines have yet been approved for use in humans in the United States and Europe.

The published data warrant cautious optimism. The high levels of neutralizing antibodies induced by these vaccine candidates suggest effectiveness (despite waning antibody titers, which is expected from a physiological immune response), yet they do not ensure that the vaccines will confer sterilizing immunity or protection from severe disease, nor that the beneficial effects will last. The vaccines eliciting substantial T-cell activity may help elevate protective immunity. Because they are soon to be tested in tens of thousands of people being enrolled in phase III trials, serious adverse events in a small percentage of people are a possibility; how frequently they occur will be consequential to the fate of each vaccine and will affect the degree to which the vaccines will be adopted by the fraction of the population who are susceptible to unscientific anti-vaccine arguments. Data from the later trials will also be useful when determining correlates of protection, which are likely to vary with age and be associated with the levels and specificity of antibodies, B cells or T cells (or combinations of these) raised by the vaccine.

The ongoing manufacturing set-up and scale-up efforts promise hundreds of millions of doses ready to be distributed by early 2021 for any of the early candidate 
vaccines, if approved by regulatory agencies. The all-hands-on-deck attitude of vaccine developers in academia, industry and government, and the advanced buy-in from government agencies, is massively reducing the typical vaccine-development timeframes. They are fast, and fit for the times.
Published online: 5 August 2020

https://doi.org/10.1038/s41551-020-00605-9

References

1. Pardi, N., Hogan, M. J., Porter, F. W. \& Weissman, D. Nat. Rev. Drug Discov. 17, 261-279 (2018).

2. Mulligan, M. J. et al. Preprint at https://doi. org/10.1101/2020.06.30.20142570 (2020)
3. Sahin, U. et al. Preprint at https://doi. org/10.1101/2020.07.17.20140533 (2020).

4. Jackson, L. A. et al. N. Engl. J. Med. https://doi.org/10.1056/ NEJMoa2022483 (2020).

5. Folegatti, P. M. et al. Lancet https://doi.org/10.1016/S01406736(20)31604-4 (2020).

6. Zhu, F.-C. et al. Lancet https://doi.org/10.1016/S01406736(20)31605-6 (2020). 